Ann. Biol. anim. Bioch. Biophys., 1978, 18 (3), 663-679

\title{
Les effets de la quantité et de la qualité des protéines du régime sur la croissance, la composition corporelle et la variation des activités de certaines enzymes du métabolisme du glucose chez le rat en croissance
}

\author{
par J. PERET, J. COTA, M. CHANEZ, Brigitte BOIS-JOYEUX
}

Centre de Recherches sur la Nutrition du C. N. R. S.

9, rue Jules-Hetzel, 92190 Meudon-Bellevue, France.

Summary. Dietary proteins in growing rat. Effects of quantity and quality on growth, body composition and variation in certain enzyme activities on glucose metabolism.

Weanling rats were fed a diet for 8 days containing various levels (10 to 70 p. 100) and qualities (egg yolk, casein, wheat gluten) of protein. Variations in liver enzyme activities of pyruvate kinase (PK), glucose-6-phosphate dehydrogenase (G6P-DH), malic enzyme (ME) and phosphoenolpyruvate carboxykinase (PEP-CK) were studied. Changes in enzymatic activity were also compared with modifications in food intake, body weight gain, nitrogen retention and apparent lipid synthesis. Increasing the protein level produced a progressive decline in ME and PK activities. The decrease in PK activity was accentuated when the biological value of dietary proteins was higher. G6P-DH activity increased with the protein fevel in rats fed with casein, and to a lesser extent when they were fed with wheat gluten. However, enzyme activity decreased slightly when the dietary egg yolk level was augmented. On the other hand, PEP-CK activity rose concomitantly with the portein level. In regard to protein intake, the relationship between the two opposite enzyme activities, PK and PEP-CK, showed that glycolysis-gluconeogenesis balance was obtained with a different intake of each protein studied : $18.2,23.7$ and $34.6 \mathrm{~g}$ of protein/day/body weight ${ }^{75}$, respectively, for egg yolk, casein and wheat gluten. This equilibrium represented the maximum daily weight gain ( $7.0 \mathrm{~g}$ for egg yolk and casein $; 5.9 \mathrm{~g}$ for wheat gluten), maximum daily protein retention $(1.07 \mathrm{~g}$ for egg yolk and casein ; $0.85 \mathrm{~g}$ for wheat gluten) and variable apparent lipid synthesis, expressed as the per cent of $\mathrm{kcal}$ of lipid deposited in relation to the total kcal deposited (36 p. 100 for egg yolk and casein ; 50 p. 100 for wheat gluten). Furthermore, at the point of intersection between glycolysis and gluconeogenesis, the daily consumption of carbohydrate energy per body weight .75 was similar for egg yolk and casein $(115$ to $120 \mathrm{kcal})$, but much lower for wheat gluten $(55 \mathrm{kcal})$. These results confirm our previous observations on young adult rats indicating that this experimental method can be used to simultaneously assess the protein and carbohydrate requirements ensuring the best weight gain, nitrogen refention and apparent lipid synthesis.

\section{Introduction.}

De nombreux auteurs ont étudié les effets des régimes riches en protéines sur l'activité des différentes enzymes (Freedland et Szepesi, 1971). Cependant, dans la majorité des cas, les résultats ont été obtenus à partir d'expériences de courte durée, 
la caséine étant généralement le support protéique du régime. En outre, malgré l'imporfance du glucose en tant que fournisseur privilégié d'ATP, dans la synthèse protéique, il manque à notre connaissance des études spécifiques concernant les relations pouvant exister entre la qualité et la quantité des protéines du régime d'une part, et le métabolisme du glucose d'autre part.

Dans un travail précédent (Peret et al., 1975) nous avons étudié l'influence de la qualité et de la quantité des protéines du régime sur l'activité de certaines enzymes du métabolisme du glucose. Les résultats obtenus ont montré que, pour une protéine donnée, le gain pondéral maximum dépend, entre autres facteurs, de l'équilibre métabolique entre la glycolyse et la néoglucogenèse. En partant de ces données, nous avons pu déterminer simultanément les besoins en protéines et en glucose, capables d'assurer aux animaux des performances maximales. Ces études ont été effectuées sur des rats adultes. Il était important de savoir si la méthode expérimentale utilisée pouvait être également appliquée à des rats jeunes. L'exposé des résultats obtenus constitue l'objet du présent travail.

\section{Matériel et méthodes.}

Animaux et protocole expérimental. - Cent quarante-trois rats mâles Wistar CF pris au sevrage sont placés dans des cages individuelles et maintenus dans des conditions contrôlées de température $\left(22 \pm 2^{\circ} \mathrm{C}\right)$ et d'éclairage $(12 / 12$ lumière de $07: 00$ à 19 : 00). Après avoir reçu ad libifum pendant 6 jours un régime préexpérimental à 10 p. 100 de protéines (caséine), ils sont répartis en quatre groupes. Le premier ( 8 rats) est sacrifié ef la composition corporelle des animaux déterminée. Les trois autres sont nourris ad libitum pendant 8 jours à l'aide des régimes contenant du jaune d'œuf, de la caséine ou du gluten de blé. Chacun de ces groupes est divisé à son tour en 7 sous-groupes (6-7 rats) nourris avec des régimes à différentes teneurs (10 à 70 p. 100) de ces protéines. A la fin de la période expérimentale, les rats sont sacrifiés par décapitation (entre 09 et $10 \mathrm{~h}$ ) et leur composition corporelle (sans sang et foie) déterminée. La composition du gain de poids est estimée par différence entre la composition corporelle finale des animaux et celle du groupe de contrôle sacrifié au début de

\section{TABLEAU 1}

Composition de la caséine, du gluten de blé et du jaune d'œuf

\begin{tabular}{|c|c|c|c|}
\hline & Caséine & $\begin{array}{l}\text { Gluten de } \\
\text { blé } \\
(\mathrm{g} / 100 \mathrm{~g})\end{array}$ & $\begin{array}{l}\text { Jaune } \\
\text { d'œuf }\end{array}$ \\
\hline $\begin{array}{l}\text { Protéines }(\mathrm{N} \times 6,25) \ldots \ldots \\
\text { Graisses } \ldots \ldots \ldots \ldots \\
\text { Glucides } * \ldots \ldots \ldots \ldots \\
\text { Cendres } \ldots \ldots \ldots \ldots \ldots \\
\text { Eau } \ldots \ldots \ldots \ldots \ldots \\
\text { Cellulose } \ldots \ldots \ldots \ldots\end{array}$ & $\begin{array}{r}80,3 \\
2,6 \\
3,0 \\
9,3 \\
4,5 \\
-\end{array}$ & $\begin{array}{r}80,0 \\
2,5 \\
5,0 \\
1,0 \\
10,0 \\
1,5\end{array}$ & $\begin{array}{r}73,4 \\
1,0 \\
3,8 \\
8,0 \\
13,8 \\
\end{array}$ \\
\hline
\end{tabular}

* Lactose, amidon ou glucose. 
l'expérience. Par ailleurs, les foies prélevés sont rapidement pesés, puis conservés à $-80^{\circ} \mathrm{C}$. Pendant la période expérimentale, la consommation de nourriture et le poids des animaux sont mesurés.

Régimes. - Les compositions du jaune d'œuf, de la caséine et du gluten de blé et des régimes expérimentaux sont présentées dans les tableaux 1 et 2 . L'augmentation du contenu en protéines des régimes est réalisée aux dépens du matériel glucidique (amidon et sucre) ; de ce fait leur apport énergétique est pratiquement semblable.

Méthodes. - La composition corporelle des animaux est déterminée par la méthode d'Abraham ef al., 1964. La teneur en eau des carcasses est d'abord mesurée

TABLEAU 2

Composition des régimes $\left({ }^{(}\right)(\mathrm{g} / 100 \mathrm{~g})$

Caséine

\begin{tabular}{|c|c|c|c|c|c|c|}
\hline 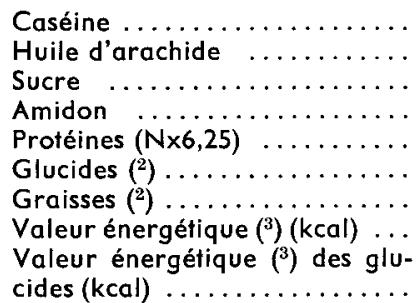 & $\begin{array}{r}12,8 \\
8,0 \\
10,0 \\
63,2 \\
10,3 \\
73,6 \\
8,3 \\
410,3\end{array}$ & $\begin{array}{r}25,0 \\
8,0 \\
10,0 \\
51,0 \\
20,1 \\
61,8 \\
8,7 \\
405,9\end{array}$ & $\begin{array}{r}37,5 \\
8,0 \\
10,0 \\
38,5 \\
30,1 \\
49,6 \\
9,0 \\
399,8\end{array}$ & $\begin{array}{r}49,7 \\
8,0 \\
10,0 \\
26,3 \\
39,9 \\
37,8 \\
9,3 \\
394,5\end{array}$ & $\begin{array}{r}61,6 \\
8,0 \\
10,0 \\
14,4 \\
49,5 \\
26,3 \\
9,6 \\
389,6\end{array}$ & $\begin{array}{r}74,1 \\
8,0 \\
10,0 \\
1,9 \\
59,5 \\
14,1 \\
9,9 \\
383,5\end{array}$ \\
\hline
\end{tabular}

Gluten de blé

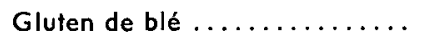

Huile d'arachide $\ldots \ldots \ldots \ldots$.

Sucre $\ldots \ldots \ldots \ldots \ldots \ldots \ldots$

Amidon $\ldots \ldots \ldots \ldots \ldots \ldots$

Protéines $(\mathrm{N} \times 6,25) \ldots \ldots \ldots \ldots$

Glucides $\left({ }^{2}\right) \ldots \ldots \ldots \ldots \ldots \ldots$

Graisses $\left(^{2}\right) \ldots \ldots \ldots \ldots \ldots \ldots$

Valeur énergétique $\left({ }^{3}\right)$ des gluci-

des (kcal) ......................

Joune d'ouf

Jaune d'œuf ..............

Huile d'arachide...$\ldots \ldots \ldots$

Sucre $\ldots \ldots \ldots \ldots \ldots \ldots \ldots$

Amidon $\ldots \ldots \ldots \ldots \ldots \ldots$

Protéines $(\mathrm{N} \times 6,25) \ldots \ldots \ldots \ldots$

Glucides $\left({ }^{2}\right) \ldots \ldots \ldots \ldots \ldots \ldots \ldots$

Valeur énergétique $\left({ }^{3}\right)$ (kcal) ...

Valeur énergétique $\left({ }^{3}\right)$ des gluci-

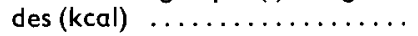

$\begin{array}{rrrrrrr}12,9 & 25,5 & 37,3 & 50,0 & 61,1 & 76,0 & 86,0 \\ 8,0 & 8,0 & 8,0 & 8,0 & 8,0 & 8,0 & 8,0 \\ 10,0 & 10,0 & 10,0 & 10,0 & 10,0 & 10,0 & - \\ 63,1 & 50,5 & 38,7 & 26,0 & 14,9 & - & - \\ 10,3 & 20,4 & 29,8 & 40,0 & 48,9 & 60,8 & 68,8 \\ 73,8 & 61,9 & 50,6 & 38,5 & 28,0 & 13,8 & 4,3 \\ 8,3 & 8,6 & 8,9 & 9,3 & 9,5 & 9,9 & 10,2 \\ 411,1 & 406,6 & 401,7 & 397,7 & 393,1 & 387,5 & 384,2 \\ 295,2 & 247,6 & 202,4 & 154,0 & 112,0 & 55,2 & 17,2\end{array}$

$\begin{array}{rrrrrrr}13,6 & 28,2 & 39,4 & 49,3 & 61,0 & 73,7 & 86,4 \\ 8,0 & 10,2 & 12,0 & 8,0 & 8,0 & 8,0 & 7,6 \\ 10,0 & 10,0 & 10,0 & 10,0 & 10,0 & 10,0 & - \\ 62,4 & 45,6 & 32,8 & 26,7 & 15,0 & 2,3 & - \\ 10,0 & 20,7 & 28,9 & 36,2 & 44,8 & 54,1 & 63,4 \\ 72,9 & 56,7 & 44,3 & 38,6 & 27,3 & 15,1 & 3,3 \\ 8,1 & 10,5 & 12,4 & 8,5 & 8,6 & 8,7 & 8,5 \\ 404,5 & 404,1 & 404,4 & 375,7 & 365,8 & 355,1 & 343,3 \\ 291,6 & 226,8 & 177,2 & 154,4 & 109,2 & 60,4 & 13,2\end{array}$

(1) Tous les régimes contiennent $(\mathrm{g} / 100 \mathrm{~g})$ : sels minéraux (Peret ef al., 1973) 4, 0 ; mélange vitaminique (Peret et al., 1973) 1, 0 et cellulose en poudre 1, $0 ;\left({ }^{2}\right)$ Dans le calcul nous avons pris en considération les graisses et les glucides contenus dans les matières de base ; $\left({ }^{3}\right)$ La valeur énergétique a été calculée en appliquant les coefficients 4,9 et $4 \mathrm{kcal}$, respectivement pour les protéines, graisses ef glucides. 


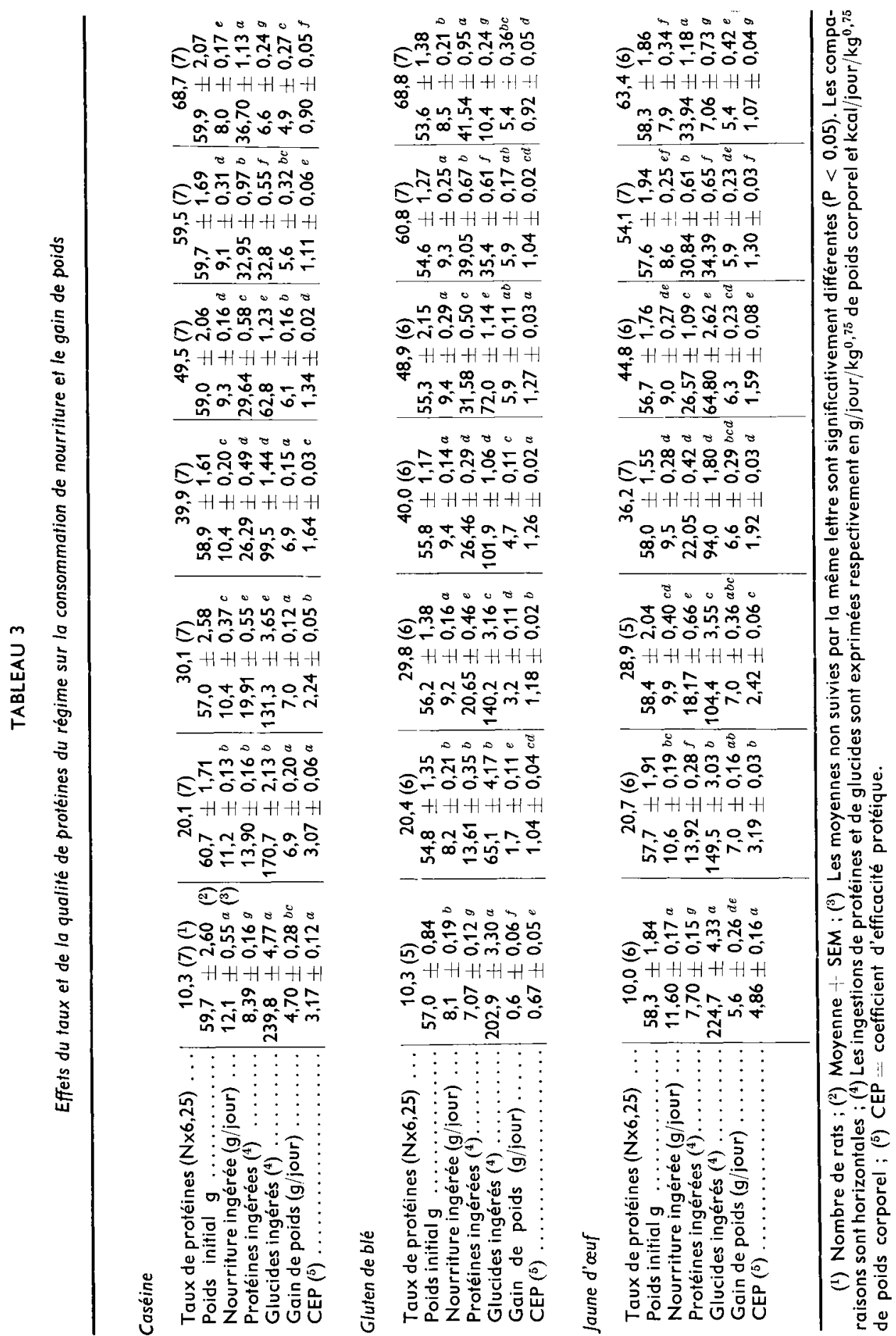


par lyophilisation. Puis, après une hydrolyse acide, les graisses sont déterminées sur l'hydrolysat par extraction en continu à l'éther de pétrole. A la fin, l'azote est dosé sur l'hydrolysat délipidé (Kjeldhal).

Les activités enzymatiques sont mesurées sur le surnageant obtenu par centrifugation à $50000 \times \mathrm{g}$ d'un homogénat de foie dans le saccharose $0,25 \mathrm{M}$. Les activités de la pyruvate kinase (EC 2.7.1.40) (PK), de l'enzyme malique (EC 1.1.1.40) (EM) et de la glucose-6-phosphate déshydrogénase (EC 1.1.1.49) (G6P-DH) sont déterminées respectivement par les méthodes de Weber ef al. (1965). Hsu et Lardy (1969) et Fitch et al. (1959). La libération du $\mathrm{NAD}^{+}$ou du NADPH est mesurée à $37^{\circ} \mathrm{C}$ et à $340 \mathrm{~nm}$ à l'aide d'un spectrophotomètre Gilford $300 \mathrm{~N}$. L'activité de la phosphoénolpyruvate carboxykinase (EC 4.1.1.32) (PEP-CK) est mesurée à $37{ }^{\circ} \mathrm{C}$, selon Chang et Lane (1966), la fixation du ${ }^{14} \mathrm{C}$ étant déterminée à l'aide d'un compteur à scintillation Packard-Tricarb. Les activités enzymatiques sont exprimées en micromoles de substrat converti à $37{ }^{\circ} \mathrm{C}$ par minute, par foie total et $100 \mathrm{~g}$ de poids corporel.

L'analyse statistique des résultats (analyse de variance et covariance, droites de regression, et tests de Tukey, Hartley et Keuls) est effectuée selon Snedecor et Coch$\operatorname{ran}(1967)$.

\section{Résultats.}

1. Consommation de nourriture et gain de poids. Les effets du taux et de la qualité des protéines du régime sur la consommation de nourriture et le gain de poids sont rapportés dans le tableau 3 .

Alors que chez les rats nourris à l'aide des régimes contenant le jaune d'œuf ou la caséine, la consommation de nourriture baisse avec l'augmentation du taux protéique, celle des animaux qui ont reçu le gluten de blé augmente, pour se maintenir constante avec des régimes compris entre 30 et 60 p. 100 de protéines.

Les animaux consommant le jaune d'œuf ou la caséine ont un gain pondéral maximum semblable, $7 \mathrm{~g} /$ jour, qui s'observe dès que la teneur en protéines du régime atteint 20 p. 100 . Au-delà d'un taux protéique de 36 p. 100 (jaune d'œuf) et de 40 p. 100 (caséine) le gain de poids commence à diminuer et cette baisse est d'autant plus marquée que la teneur en protéines du régime est plus élevée. Les rats nourris avec le gluten de blé ne réalisent qu'un gain pondéral quotidien maximum de $5,9 \mathrm{~g}$ et cela, malgré la consommation de régimes contenant des taux protéiques fort élevés (entre $48,9$ et 68,8 p. 100$)$.

Le coefficient d'efficacité protéique (CEP) des animaux recevant le jaune d'œuf et la caséine diminue avec l'augmentation du taux protéique. Par contre chez les rats nourris avec le gluten de blé, le CEP augmente avec le taux protéique. Les valeurs les plus élevées s'observant lorsque le régime contient entre 40 et 50 p. 100 de protéines. C'est seulement au-delà de ces niveaux protéiques qu'il commence à baisser.

2. Composition du gain de poids. Les variations de la composition corporelle et du gain de poids des animaux figurent dans les tableaux 4,5 et 6 . 


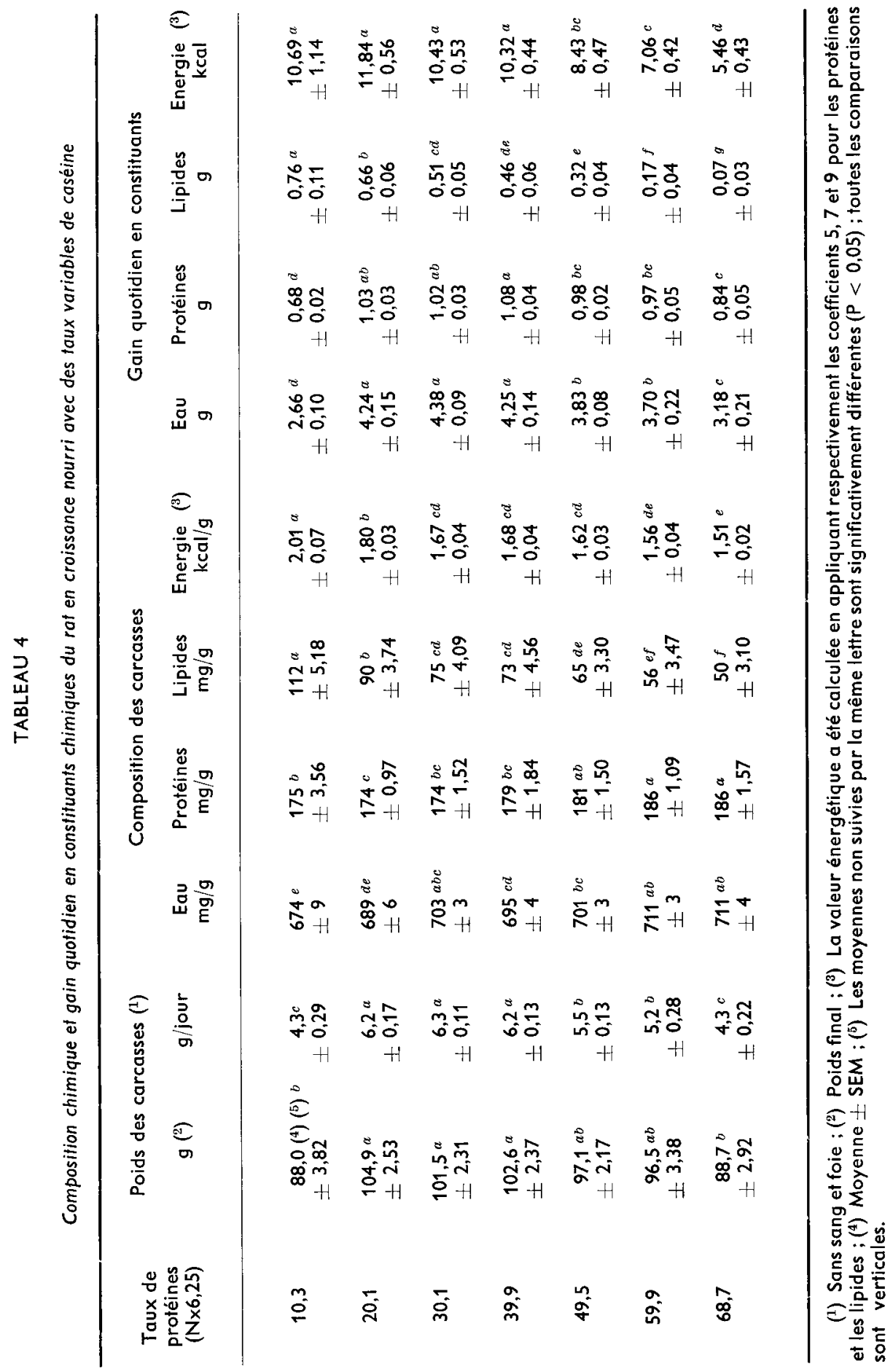




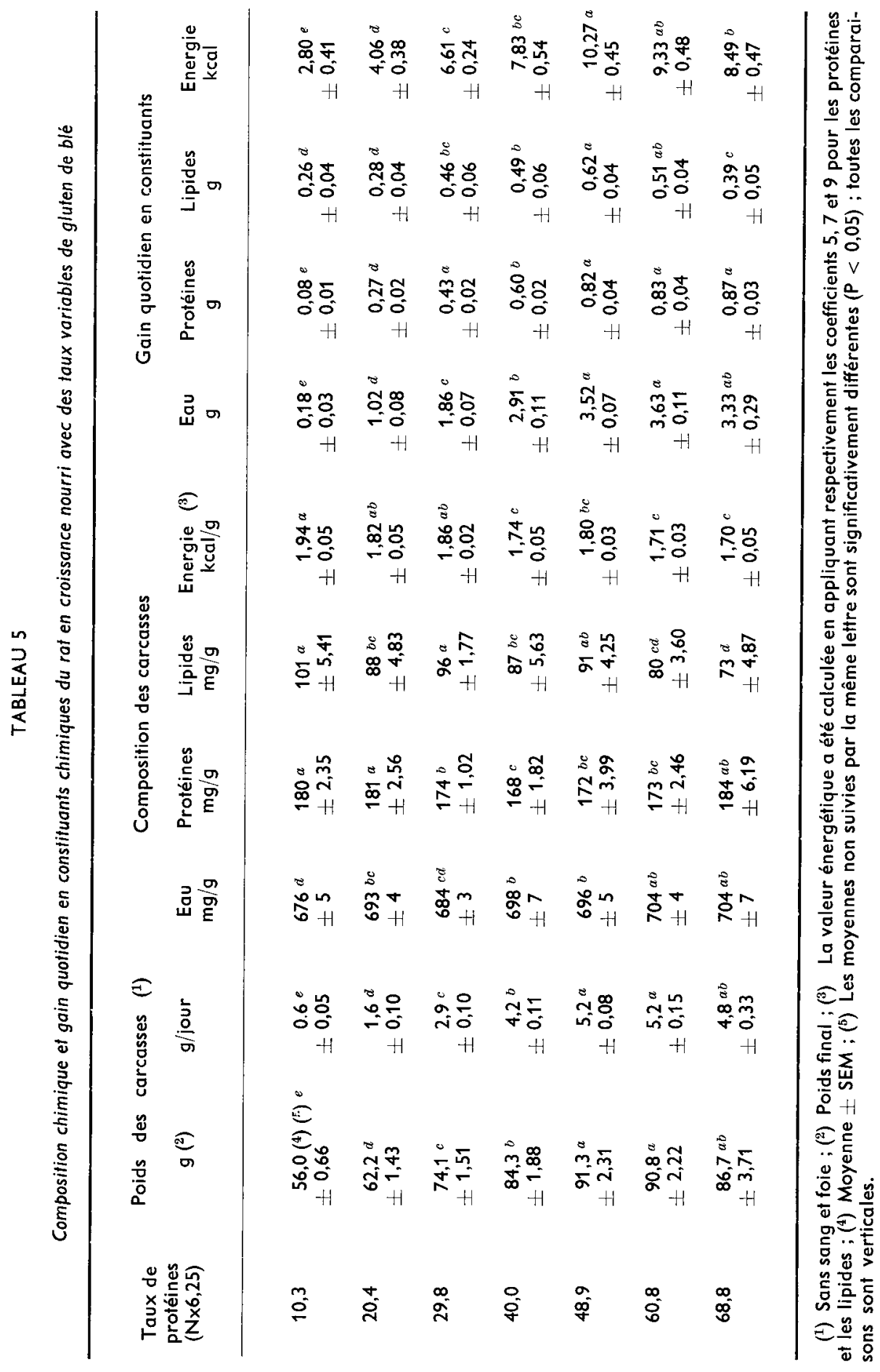




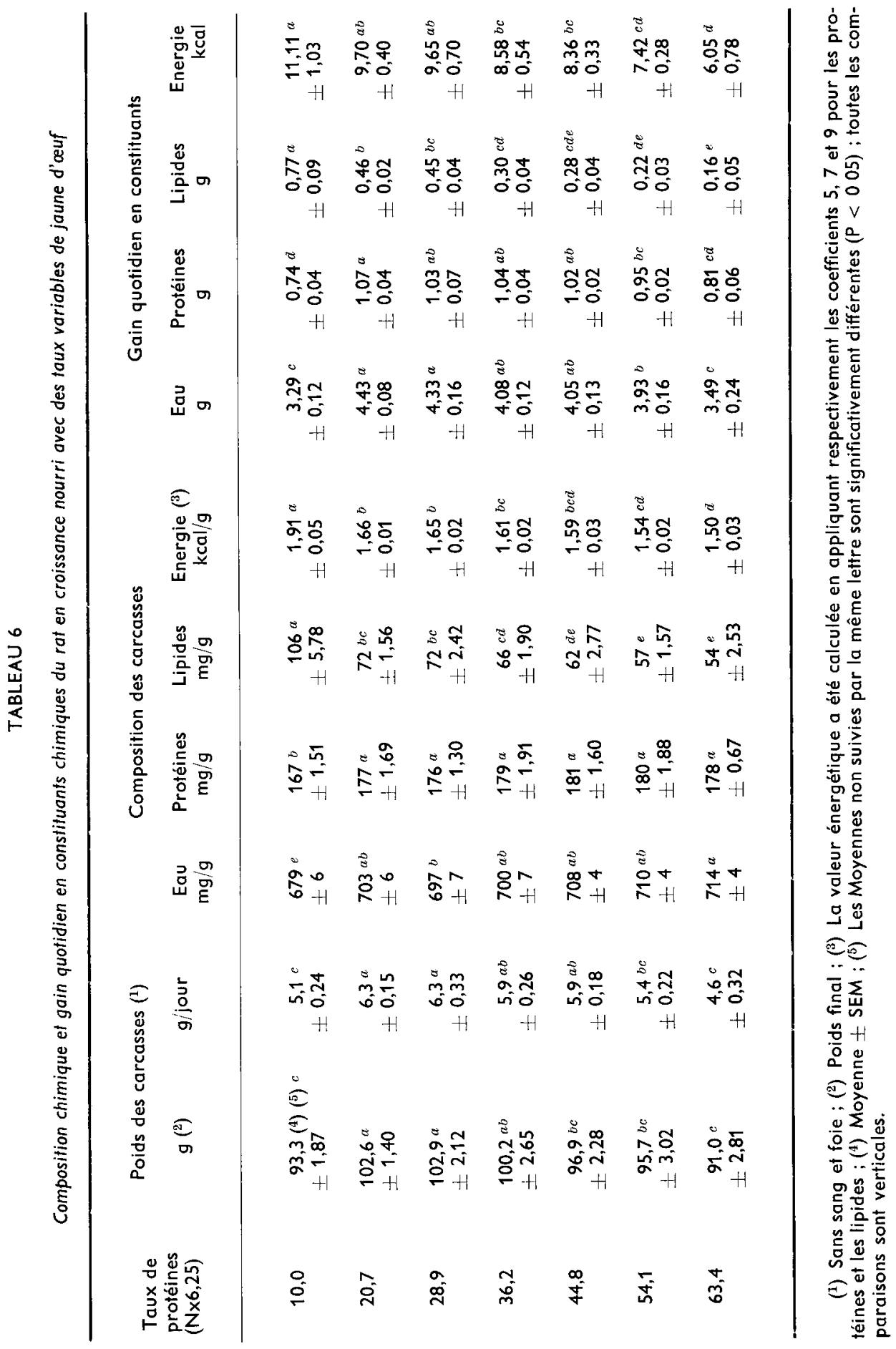


Alors que les rats nourris avec le jaune d'œuf et la caséine ont une rétention azotée maximum de $1,07 \mathrm{~g}$ protéines/jour, ceux qui reçoivent le gluten de blé ne retiennent que 0,82 à $0,87 \mathrm{~g}$ protéines/jour. Par ailleurs, on n'observe pas de relation étroite entre l'évolution du gain de poids et la rétention azotée : la diminution du gain de poids avec l'augmentation du taux protéique du régime précède celle de la rétention azotée. Cependant chez les rats nourris avec le gluten de blé, la rétention azotée ne diminue pas, même quand les animaux consomment un régime à 68,8 p. 100 de protéines.

Chez les rats nourris au jaune d'œuf et à la caséine, le gain journalier de lipides diminue au fur et à mesure que le taux protéique du régime augmente. Par contre, chez les animaux qui ont consommé le gluten de blé, le gain de lipides augmente avec le taux protéique jusqu'à une concentration de 48,9 p. 100 de protéines dans le régime. Par la suite, le gain de lipides commence à diminuer, sans łoutefois atteindre des valeurs comparables à celles des rats recevant les régimes à base de jaune d'œuf ou de caséine.

3. Activités enzymatiques. Les variations des activités enzymatiques en relation avec le taux et la qualité des protéines du régime sont présentées dans la figure 1.

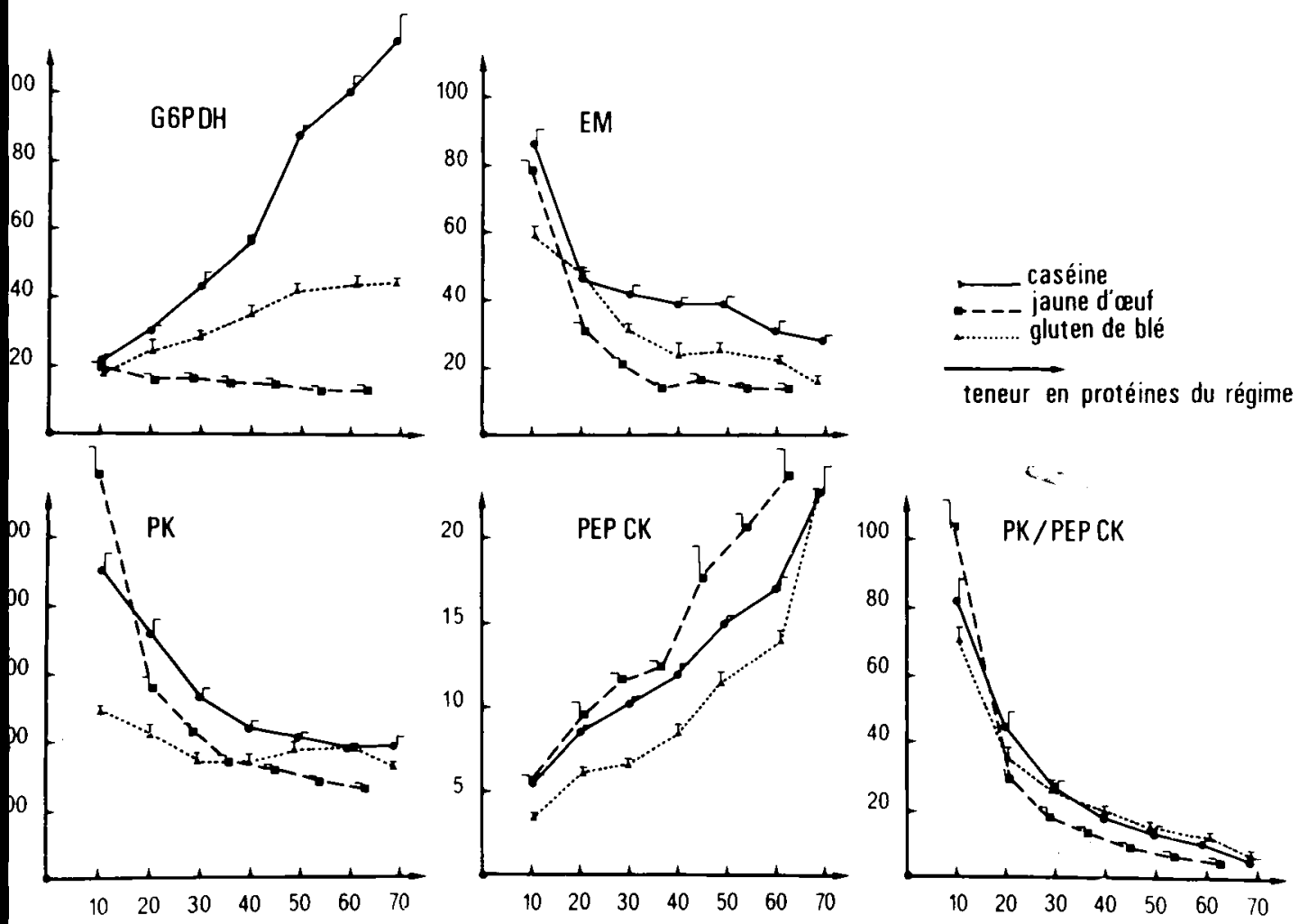

FIG. 1. - Taux et qualité des protéines du régime et variations des activités hépatiques de la G6P-DH, de I'EM, de la PK ef de la PEP.CK. Les activités enzymatiques sont exprimées en micromoles de substrat converti à $37^{\circ} \mathrm{C}$ par minute par foie total ef $100 \mathrm{~g}$ de poids corporel. Les barres représentent \pm SEM. 
Les activités des enzymes qui libèrent le NADPH, l'enzyme malique (EM) ef la G6P-DH ne varient pas dans le même sens. Ainsi, quelle que soit la protéine mise en œuvre, l'activité de l'EM diminue avec l'augmentation du taux protéique du régime. Par contre, tandis que chez les rats nourris avec le jaune d'œuf l'activité de la G6PDH décroît légèrement avec l'augmentation du taux protéique, chez les rats recevant le gluten de blé et la caséine elle s'accroît ; l'augmentation de l'activité de l'enzyme étant importante dans le dernier cas.

Les activités de la PEP-CK (néoglucogenèse) et de la PK (glycolyse) varient en sens contraire. Au fur et à mesure que l'ingestion de protéines augmente, l'activité de la PEP-CK s'accroît. Ce phénomène ne semble pas influencé par la qualité des protéines alimentaires et l'augmentation de l'activité de l'enzyme en fonction du taux protéique du régime est pratiquement semblable.

Par contre, nos résultats indiquent que la baisse de l'activité de la PK en fonction du taux protéique du régime semble varier avec la qualité des protéines étudiées. Elle est d'autant plus importante que la qualité de la protéine est meilleure : jaune d'œuf $>$ caséine $>$ gluten de blé.

Le résultat des variations en sens opposé de la PK et de la PEP.CK est la baisse du rapport PK/PEP-CK avec le taux protéique.

4. L'équilibre entre la glycolyse ef la néoglucogenèse et ses relations avec le gain de poids et sa composition.

Pour éfudier ces aspects, nous avons dans un premier temps calculé les activités relatives de la $\mathrm{PK}$ et de la PEP-CK en les exprimant en pourcentage de leur activité maximum (coefficient 100) correspondant à un taux protéique de 10 p. 100 (PK) et de 60-70 p. 100 (PEP-CK). Puis, dans un second temps, nous avons calculé les variations de ces deux enzymes en fonction de la consommation journalière de protéines rapportée au poids métabolique de l'animal $\left(\mathrm{P}^{0}, 75\right)$. Sont ainsi obtenues les valeurs des points où les activités opposées de la PK et de la PEP-CK se croisent pour chacune des protéines étudiées (fig. 2). Par la suite, nous avons comparé les points d'intersection avec le gain pondéral des animaux (fig. 2) et calculé pour ces mêmes points, la consommation quotidienne de glucose, en l'exprimant en $\mathrm{kcal} / \mathrm{jour} /$ poids corporel ${ }^{0,75}$. Enfin, pour rendre compte des éventuelles relations qui peuvent exister entre la glycolyse et la néoglucogenèse d'une part, et la synthèse apparente de lipides d'autre part, nous avons calculé pour les mêmes points d'intersection les kcal d'origine lipidique retenues par jour, exprimées en pourcentage des kcal totales.

Les résultats obtenus indiquent que les points d'intersection varient d'une protéine à l'autre : $18,18 \mathrm{~g}, 23,71 \mathrm{~g}$ et $34,57 \mathrm{~g}$ de protéines ingérées/jour/poids corpore/0,75 respectivement pour le jaune d'œuf, la caséine et le gluten de blé (fig. 2). Ces points correspondent approximativement au gain pondéral maximum des animaux d'expérience : $7 \mathrm{~g} /$ jour pour les rats nourris avec le jaune d'œuf et la caséine et $5,9 \mathrm{~g} / \mathrm{jour}$ pour ceux qui ont reçu le gluten de blé. Au-delà des points d'intersection, lorsque la néoglucogenèse prend le pas sur la glycolyse, le gain de poids commence à baisser.

En outre, bien que les points d'intersection des droites de régression soient différents pour les animaux nourris avec le jaune d'œuf et la caséine, la consommation quotidienne de glucose, exprimée en $\mathrm{kcal} / \mathrm{jour} /$ poids corporel $\mathrm{l}^{0,75}$ est semblable : 
entre 115 et $120 \mathrm{kcal}$. Par contre, chez les animaux recevant le gluten de blé, la consommation de glucose est plus faible, autour de $55 \mathrm{kcal} /$ jour $/$ poids corpore ${ }^{0.75}$.

D'autre part, il faut remarquer que les animaux nourris avec le jaune d'œuf et la caséine ont aux points d'intersection respectifs, la même synthèse apparente de lipides : 36 p. 100 de kcal retenues par jour sont d'origine lipidique. Les rats qui ont consommé le gluten de blé sont plus gras, puisque $50 \mathrm{p}$. 100 des kcal retenues par jour sont de nature lipidique.

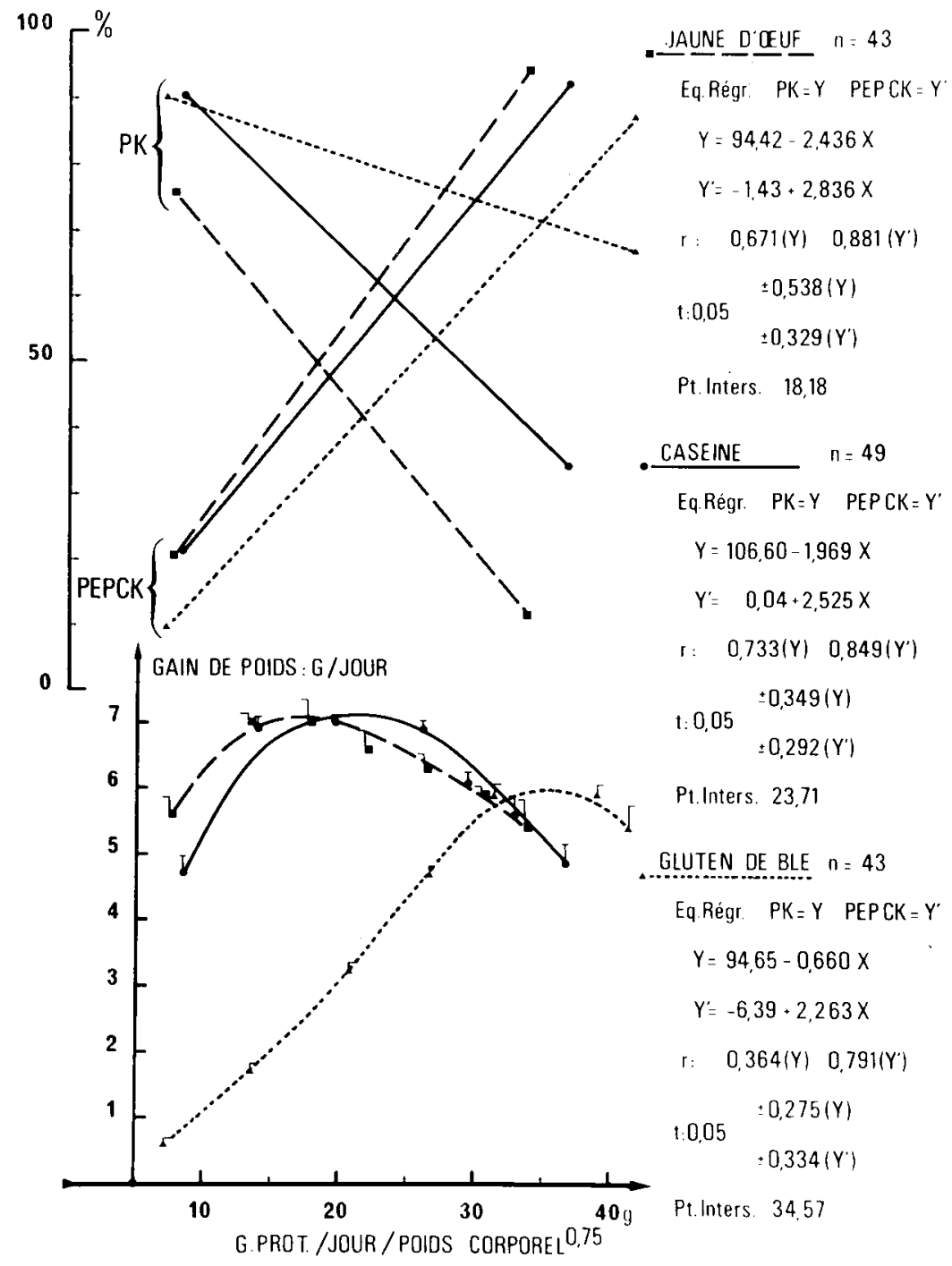

FIG. 2. - Les activités relatives de la PK et de la PEP-CK en fonction de la consommation de protéines par jour et poids corpore/ ${ }^{0,75}$. Chaque point représente la moyenne de 5 à 7 animaux (voir tabl. 3). Les barres représentent \pm SEM. 


\section{Discussion.}

\section{Consommation de nourriture ef gain de poids.}

Les résultats concernant les effets du taux el de la qualité des protéines du régime sur la consommation de nourriture et le gain de poids confirment les observations de la littérature (Barnes ef al., 1946 ; Hegsted et Chang, 1965 ; Mauron ef al., 1973 ; Peng et al., 1974 ; Peret et al., 1975). La consommation de nourriture est accrue lorsque la concentration du régime en protéines de faible qualité est augmentée et inversement, elle est diminuée quand le taux protéique du régime est élevé et l'apport protéique de bonne qualité. Ainsi, le gain de poids maximum (5,9 $\mathrm{g} /$ jour) est atteint chez les animaux nourris avec le gluten de blé (facteurs limitants : lysine ef thréonine) par l'augmentation de leur consommation, celle-ci pouvant couvrir en partie leurs besoins en acides aminés essentiels limitants. Néanmoins, contrairement à ce qu'on observe chez les rats adultes (Peret et al., 1975) les rats en croissance nourris avec le gluten de blé sont incapables de réaliser un gain pondéral semblable à celui des rats qui ont reçu le jaune d'œuf ou la caséine. Au-delà d'une concentration en protéines optimum pour la croissance, la consommation de nourriture et le gain de poids sont diminués, puisque le régime n'est plus capable d'assurer une couverture énergétique satisfaisante (Munro, 1964).

\section{Composition du gain de poids.}

Ces observations sont en grande partie confirmées par les variations de la composition corporelle et du gain de poids. La non-concordance entre l'évolution du gain de poids et la rétention azotée s'explique par la variation en sens inverse du taux protéique du régime et de la synthèse apparente de lipides; celle-ci diminuant au fur et à mesure que le taux protéique du régime augmente. Il apparaît donc, que la baisse de l'apport de glucose (par élévation du taux protéique) agit avant tout sur la lipogenèse, en la diminuant (Peret ef al., 1977 résultats non publiés), le glucose étant utilisé en priorité pour fournir l'énergie nécessaire à la synthèse de protéines. Il s'ensuit que le gain de poids souvent considéré comme « indicateur » de la valeur nutritionnelle d'un régime donné, ne représente qu'une notion relative. Il peuł avoir un caractère représentatif, seulement quand l'apport protéique (acides aminés essentiels) et énergétique (glucose) du régime sont capables d'assurer une rétention azotée ef une synthèse apparente de lipides maximum (Allison, 1964 ; Munro, 1964 ; Rérat, 1971 ; Jacquot et Peret, 1972 ; Cooke ef al., 1972 ; Hartsook ef al., 1973).

\section{Activités enzymatiques.}

Chez les rats nourris avec la caséine et le gluten de blé, les variations de l'activité des enzymes qui libèrent NADPH sont semblables à celles de la littérature. L'activité de la G6P-DH augmente avec la teneur en protéines (Schimke, 1962 ; Vaughan et Winders, 1964 ; Peret et al., 1975) alors que celle de l'enzyme malique diminue (Vaughan et Winders, 1964 ; Cohen et Teitelbaum, 1966 ; Szepesi et Freedland, 1967 ; Yeh et Leveille, 1969 ; Peret et al., 1975). Bien que l'augmentation de l'activité de la G6P-DH soit beaucoup plus importante avec la caséine qu'avec le gluten de blé, elle 
ne semble pas liée à la qualité nutritionnelle de ces protéines : — l'induction de l'enzyme ne dépend pas de l'ingestion d'un profil spécifique d'acides aminés (Taketa et al., 1972, 1973) et - l'activité de l'enzyme n'est pas augmentée par la supplémentation du gluten de blé avec ses acides aminés limitants (Carrozza ef al., 1974 ; Livrea et al., 1974). En outre, chez les rats nourris avec le jaune d'œuf, l'activité de l'enzyme, au lieu de s'accroître, diminue légèrement. II apparaît donc que l'augmentation de l'activité de la G6P-DH avec le taux protéique qui s'observe chez les rats qui ont reçu la caséine n'est pas spécifique de l'apport protéique du régime ; elle est probablement due à d'autres facteurs propres à la caséine utilisée (caséine lactique, non purifiée) qui restent à déterminer.

La baisse de l'activité de l'EM avec l'augmentation du taux protéique peut être due à la diminution de l'apport de glucose, celui-ci étant un des inducteurs spécifiques de l'enzyme (Vaughan et Winders, 1964 ; Leveille et Yeh, 1972 ; Frenkel ef al., 1972 ; Romsos et Leveille, 1974) soit au résultat d'un effet répresseur produit par l'accumulation de pyruvate provenant de l'intensification des transaminations. La concomittance de ces deux processus n'est pas exclue.

La principale observation de ce travail est la relation inverse entre l'activité de la PEP-CK (néoglucogenèse) qui augmente, et celle de la PK (glycolyse) qui diminue lorsque le faux protéique du régime augmente. Le parallélisme entre l'augmentation de l'activité de la PEP-CK et celle de l'ingestion de protéines témoigne de l'intensification du processus de néoglucogenèse. La diminution de l'activité de la PK avec l'augmentation de la consommation de protéines a été observée par différents auteurs (Krebs et Eggleston, 1965 ; Freedland et al., 1966 ; Szepesi et Freedland, 1967). Cependant, nos résuliats indiquent que la baisse de l'activité de la PK varie aussi selon la qualité des protéines du régime. Ces observations sont semblables à celles effectuées chez des rats adultes (Peret et al., 1975) ; on pourrait les expliquer par l'interférence entre deux processus : a) l'utilisation et la production de l'ATP nécessaire pour la synthèse des protéines, et ; b) les mécanismes de régulation de la PK et surtout de la glycolyse et de la néoglucogenèse.

a) En dehors d'un apport quantitatif et qualitatif optimal d'acides aminés, la synthèse de protéines nécessite un nombre important de molécules d'ATP (ou GTP) (Haselkorn ef Rothman-Denes, 1973 ; Hegsted, 1974 ; Buttery et Boorman, 1976 ; Campbell, 1977). Il est bien évident que la synthèse de protéines est d'autant plus intense, que le régime fournit une quantité suffisante et équilibrée d'acides aminés essentiels, comme c'est le cas lorsque l'apport protéique est de bonne qualité. On peut donc déduire que dans ces conditions les besoins en ATP vont être accrus. Par ailleurs, le glucose reste le meilleur substrat fournisseur d'énergie, puisque 1 mole de glucose fournit 38 moles d'ATP.

b) Le phosphoénolpyruvate (PEP), substrat de la PK, est siłué à un des carrefours essentiels du méłabolisme intermédiaire. Chez les animaux ayant une consommation élevée de glucose, le PEP obtenu par la glycolyse est transformé en pyruvate par la PK. Celui-ci, sous l'action de la pyruvate déshydrogénase, est métabolisé en acétyl-CoA, qui peut être oxydé (cycle tricarboxylique) ou utilisé pour la synthèse des acides gras. Lorsque la consommation de glucose est faible (cas des régimes hyperprotéiques), le PEP est obtenu à partir du pyruvate ou de l'oxaloacétate par néoglucogenèse sous 
l'action de la pyruvate carboxylase et de la PEP-CK. Cependant, la voie de la glycolyse étant prédominante (Weber ef al., 1967 ; Weber, 1969) le PEP formé est recyclé en pyruvate (Friedman et al., 1971 ; Kramer et Freedland, 1972 ; Hers, 1976). Le processus de néoglucogenèse ne peut se dérouler d'une manière satisfaisante (PEP $\rightarrow$ 2-phosphoglycérate $\rightarrow$ glucose) que lorsque l'activité de la PK est inhibée (Weber et al., 1967 ; Weber, 1969). La glycolyse libère 2 moles d'ATP, jusqu'au stade du pyruvate alors que la synthèse de glucose à partir de celui-ci via l'oxaloacétate(néoglucogenèse) en exige 6 moles; il en résulte un déficit de 4 moles d'ATP. Ainsi, bien que la glycolyse ef la néoglucogenèse puissent se dérouler en même temps, un de ces deux processus doit prédominer pour prévenir un gaspillage d'énergie. Lorsque la néoglucogenèse prévaut, l'ATP nécessaire pour la synthèse du glucose est obtenu par l'oxydation des acides gras (Krebs, 1966). Ces derniers fournissent aussi l'acétyl-CoA et le NADH, activateurs de la pyruvate carboxylase ef de la PEP-CK et probablement inhibiteurs de la PK (Weber, 1969 ; Hers, 1976).

Les résultats obtenus dans nos expériences justifient amplement ces explications théoriques.

Ainsi, lorsque les rats sont nourris avec le jaune d'œuf (protéine de haute valeur biologique), la synthèse protéique nécessite des quantités forf élevées d'ATP, puisque les acides aminés ingérés sont presque complètement utilisés. Si les animaux augmentent leur ingestion de protéines, la capacité de la glycolyse à fournir de l'ATP est rapidement dépassée et l'équilibre entre la glycolyse et la néoglucogenèse est rompu au profit de cette dernière. II s'ensuit rapidement une inhibition de la PK et une stimulation de l'activité de la PEP-CK. De plus, on observe une baisse du gain de poids, de la synthèse apparente des lipides et, plus tardivement, de la rétention azotée. Cela est dû à un déficit de glucose (arrêt de la lipogenèse) d'une part, et d'autre part au fait que l'ATP fourni par l'oxydation des graisses est insuffisant pour satisfaire à la fois les besoins de la synthèse de glucose et de protéines.

Des phénomènes semblables ont lieu lorsque les animaux reçoivent la caséine. Cependant, dans ce cas l'équilibre entre la glycolyse et la néoglucogenèse est atteint plus tard, puisque l'apport protéique est moins bien équilibré en acides aminés et de ce fait le besoin en ATP moins important.

Lorsque les rats sont nourris avec une protéine de faible qualité (gluten de blé) l'apport d'acides aminés est déséquilibré ef l'ATP fourni par la glycolyse est partiellement utilisé pour la synthèse protéique. Une partie de cette énergie est stockée sous forme de lipides et l'autre utilisée pour le recyclage du pyruvate. Certains acides aminés, non utilisés pour la synthèse protéique, vont fournir du PEP via pyruvate et/ou oxaloacétate. II s'ensuit que l'activité de la PK ne diminue que lentement, tandis que celle de la PEP-CK augmente proportionnellement avec les acides aminés non utilisés pour la synthèse protéique. En outre, la rétention azotée est faible et les animaux sont gras. C'est seulement lorsque l'augmentation de l'ingestion de protéines est capable de couvrir les besoins en acides aminés ef la synthèse protéique maximale, que l'ATP fourni par la glycolyse est lui aussi pleinement utilisé. Au-delà de ce niveau, l'équilibre entre la glycolyse et la néoglucogenèse est rompu, l'activité de la PK baisse sensiblement, de même que la synthèse apparente de lipides. Cependant bien que l'équilibre entre la glycolyse et la néoglucogenèse soit tardif, les rats nourris au gluten de blé ne peuvent pas réaliser un gain de poids et une rétention azotée semblables à ceux 
qui reçoivent la caséine et le jaune d'œuf. Cela s'explique par le fait que les rats au sevrage ont une croissance très rapide et un renouvellement des protéines accéléré, donc un besoin accru, à la fois en acides aminés et en ATP (Schimke, 1970 ; Brostrom et Jeffay, 1970 ; Millward ef al., 1976). Or, il est bien évident que l'augmentation excessive de l'ingestion du gluten de blé (taux élevé dans le régime) ne peut assurer à la fois la couverture optimale en acides aminés et en énergie.

Cependant l'ensemble de nos explications doit être étayé par des mesures des flux glucolytiques et néoglucogénétiques puisque les mesures des variations des activités de la PK (glycolyse) et de la PEP-CK (néoglucogenèse), dans nos conditions expérimentales, peuvent seulement indiquer les orientations du métabolisme glucidique.

En conclusion, nos résultats indiquent que pour une protéine donnée, la croissance maximum (rétention azotée et synthèse apparente de lipides maximales) dépend non seulement de sa capacité à couvrir les besoins quantitatifs et qualitatifs d'acides aminés mais aussi, d'un maximum d'énergie disponible sous forme d'ATP qui peut être mis en évidence par l'équilibre métabolique entre la glycolyse et la néoglucogenèse.

Par ailleurs, de nos expériences antérieures (Peret et al., 1975) et des observations présentées dans ce travail, il résulte que la méthode expérimentale utilisée permet, tout aussi bien chez le rat en croissance pris au sevrage, que chez le rat adulte, une appréciation simultanée des besoins en protéines et en glucose, capables d'assurer le meilleur gain de poids et, implicitement, une rétention azotée ef une synthèse apparente de lipides optimales.

Reçu en octobre 1977.

Accepté en janvier 1978.

\section{Références}

ABRAHAM J., MORIN-JOMAIN M., PERETIANU J., 1964. Nouvelle technique de détermination de la composition corporelle des animaux de laboratoire. Bull. Soc. Chim. biol., 46, 755-758.

ALLISON J. B., 1964. The nutritive value of dietary proteins, 41-86. In MUNRO H. N., ALLISON J. B., Mommalian protein metabolism. Vol. II. Acad. Press, New York-London.

BARNES R. H., BATES M. J., MAACK J. E., 1946. The growth and maintenance utilization of dietary protein. J. Nutr., 32, 535-548.

BROSTROM C. O., JEFFAY H., 1970. Protein catabolism in rat liver homogenates. A re-evaluation of the energy requirement for protein catabolism. J. biol. Chem., 245, 4001-4008.

BUTTERY P. J., BOORMAN K. N., 1976. The energetic efficiency of aminoacid metabolism, 197 206. In Protein metabolism and nutrition, Europ. Assn. Anim. Prod. Publ. no 16, Butterworth, London-Boston.

CAMPBELL P. N., 1977. Recent advances in eukariotic protein synthesis, 12-14. In Proc. 2 nd int. Sympos. Protein metabolism and nutrition, Europ. Assn. Anim. Prod. Publ. no 22. Center for Agricult. Publish. Doc. Wageningen.

CARROZZA G., LIVREA G., MANASSERI L., MURACA U., 1974. Differential effect of gluten and casein diets on rat liver HMP shunt dehydrogenases. J. Nutr., 104, 819-827.

CHANG H. C., LANE M. D., 1966. The enzymatic carboxylation of phosphoenolpyruvate. J. biol. Chem., 241, 2413-2420.

COHEN A. M., TEITELBAUM A., 1966. Effects of different levels of protein in sucrose and starch diets on lipid synthesis in the rat. Isr. J. med. Sci., 2, 727-732.

COOKE R., LODGE G. A., LEWIS D., 1972. Influence of energy and protein concentration in the diet on the performance of growing pigs. 3. Response to differences in levels of both energy and protein. Anim. Prod., 14, 219-228.

FITCH W. M., HILL R., CHAIKOFF I. L., 1959. The effect of fructose feeding on glycolytic enzyme activities of the normal rat liver. J. biol. Chem., 234, 1048-1051. 
FREEDLAND R. A., CUNLIFFE T. L., ZINKL J. G., 1966. The effect of insulin on enzyme adaptations to diets and hormones. J. biol. Chem., 241, 5448-5451.

FREEDLAND R. A., SZEPESI B., 1971. Control of enzyme activity : nutritionnal factors, 103-140. In RECHEIGL M. Jr. Enzyme synthesis and degradation in mammalian systems. Karger, Basel.

FRENKEL R., STARK M. J., STAFFORD J., 1972. Increased " malic enzyme » activity during adaptation to a low protein diet. Biochem. Biophys. Res. commun., 49, 1684-1689.

FRIEDMANN B., GOODMAN E. H., SAUNDERS H. L., 1971. An estimation of pyruvate recycling during gluconeogenesis in the perfused rat liver. Arch. Biochem. Biophys., 143, 566-578.

HARTSOOK E. W., HERSHBERGER T. V., NEE J. C. M., 1973. Effects of dietary protein content and ratio of fat to carbohydrate calories on energy metabolism and body composition of growing rats. J. Nutr., 103, 167-178.

HASELKORN R., ROTHMAN-DENES L. B., 1973. Protein synthesis. Ann. Rev. Biochem., 42, 397-438. HEGSTED D. M., 1974. Energy needs and energy utilization. Nufr. Rev., 32, 33-38.

HEGSTED D. M., CHANG YET-OY., 1965. Protein utilization in growing rats. I. Relative growth index as a bioassay procedure. J. Nutr., 85, 159-168.

HERS H. G., 1976. The usefulness of « futile cycles ». Biochem Soc. Transact., 4, 985-988.

HSU R. Y., LARDY H. A., 1969. Malic enzyme. Methods Enzymol., 17, 230-235.

JACQUOT R., PERET J., 1972. Protein efficiency ratio and related method, 317-346. In BIGWOOD J., International encyclopaedia of food and nutrition, Vol. 11, Protein and aminoacid fonstions. Pergamon Press, Oxford.

KRAMER J. W., FREEDLAND R. A., 1972. Evidence for a functional role of pyruvate kinase in decreasing gluconeogenesis in the perfused rat liver. Proc. Soc. exp. Biol. Med., 140, 1399-1401.

KREBS H. A., 1966. The regulation of the release of ketone bodies by the liver. Adv. Enzyme Regul. 4, 339-353.

KREBS H. A., EGGLESTON L. V., 1965. The role of pyruvate kinase in the regulation of gluconeogenesis. Biochem. J., 94, 3c-4c.

LEVEILLE G. A., YEH Y. Y., 1972. Influence of intermittent fasting on protein-free feeding on lipid metabolism in young cockerel. J. Nutr., 102, 733-740.

LIVREA G., CARROZZA G., MANASSERI L., MURACA U., 1974. On the repression of rat liver HMP shunt dehydrogenases by wheat gluten. J. Nutr., 104, 957-961.

MAURON J., MOTTU F., SPOHR G., 1973. Reciprocal induction and repression of serine dehydratase and phosphoglycerate dehydrogenase by proteins and dietary essential aminoacids in rat liver. Eur. J. Biochem., 32, 331-342.

MILLWARD D. J., GARLICK P. J., JAMES W. P. T., SENDER J. P., WATERLOW J. C., 1976. Protein turnover. 49-69, In Protein metabolism and nutrition. Europ. Assn. Anim. Prod. Publ. $n^{\circ} 16$, Butterworths, London-Boston.

MUNRO H. N., 1964. General aspects of the regulation of protein metabolism by diet and by hormones, 381-481, In MUNRO H. N., ALLISON J. B., Mammalian protein metabolism, Acad. Press, New York.

PENG YEH-SHAN, MELIZA L. L., VAVICH M. G., KEMMERER A. R., 1974. Changes in food intake and nitrogen metabolism of rats while adapting to a low or high protein diet. J. Nutr., 104, 1008-1017.

PERET J., MACAIRE I., CHANEZ M., 1973. Schedule of protein ingestion, nitrogen and energy utilization and circadian rhythm of hepatic glycogen, plasma corticosterone and insulin in rats. J. Nutr., 103, 866-874.

PERET J., CHANEZ M., COTA J., MACAIRE I., 1975. Effects of quantity and quality of dietary protein and variation in certain enzyme activities on glucose metabolism in the rat. J. Nutr., 105, 1525-1534.

RÉRAT A., 1971. La valeur biologique des protéines : quelques acquisitions récentes. Ann. Zootech., 20, 193-246.

ROMSOS D. R., LEVEILLE G. A., 1974. Effect of diet on activity of enzymes involved in fatty acid and cholesterol synthesis. Adv. Lipid Res., 12, 97-146.

SCHIMKE R. T., 1962. Adaptive characteristics of urea cycle enzymes in the rat. J. biol. Chem., 237, 459-468.

SCHIMKE R. T., 1970. Regulation of protein degradation in mammalian tissues, 177-228. In MUNRO H. N., Mammalian protein mefabolism, Vol. IV. Acad. Press, New York-London. 
SNEDECOR G. W., COCHRAN W. G., 1967. Statistical methods. Ed. 6th, lowa State Univ. Press, Ames, lowa, USA.

SZEPESI B., FREEDLAND R. A., 1967. Alterations in the activities of several rat liver enzymes at various times after initiation of a high protein regimen. J. Nutr., 93, 301-306.

TAKETA K., HANESHIGE Y., KOSAKA K., 1972-1973. Dual control of glucose-6-phosphate dehydrogenase induction in rat liver by dietary glucose and amino acids. Enzyme, 14, 105-116.

VAUGHAN D. A., WINDERS R. L., 1964. Effects of diet on HMP dehydrogenase and malic TPN dehydrogenase in the rat. Amer. J. Physiol., 206, 1081-1084.

WEBER G., 1969. Regulation of pyruvate kinase. Adv. Enzyme Regul., 7, 15-40.

WEBER G., STAMM N. B., FISHER E. A., 1965. Insulin : inducer of pyruvate kinase. Science, 149, 65-67.

WEBER G., LEA M. A., HIRD CONVERY J. J., STAMM N. B., 1967. Regulation of gluconeogenesis and glycolysis : studies of mechanisms controlling enzyme activity. Adv. Enzyme Regul., 5, 257-298.

YEH YU-YAN, LEVEILLE G. A., 1969. Effect of dietary profein on hepatic lipogenesis in the growing chick. J. Nutr., 98, 356-366. 